INRA Prod. Anim., 2010, 23 (1), 53-64

\title{
Évolution des performances et de la robustesse des animaux en élevage porcin
}

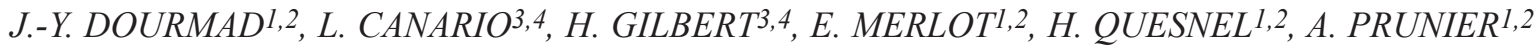 \\ ${ }^{1}$ INRA, UMR1079 Systèmes d'Elevage Nutrition Animale et Humaine, F-35590 Saint-Gilles, France \\ 2 Agrocampus Ouest, Systèmes d'Elevage Nutrition Animale et Humaine, F-35000 Rennes, France \\ ${ }^{3}$ INRA, UMR1313 Génétique Animale et Biologie Intégrative, F-78352 Jouy-en-Josas, France \\ ${ }^{4}$ AgroParisTech, Génétique Animale et Biologie Intégrative, 16 rue Claude Bernard, F-75231 Paris, France \\ Courriel : Jean-Yves.Dourmad@rennes.inra.fr
}

Au cours de ces dernières années, on a assisté à une amélioration marquée des performances dans les élevages porcins. La prolificité s'est fortement accrue et la question se pose de la capacité des truies et des porcelets à faire face aux nombreux changements biologiques qui sous-tendent cette amélioration des performances. De la même manière, on peut s'interroger sur la capacité des animaux à forte croissance musculaire à faire face aux différents challenges, en particulier sanitaires, auxquels ils sont confrontés.

Les notions de robustesse, de rusticité, de flexibilité ou de plasticité renvoient à la capacité des animaux à s'adapter à des milieux divers tout en maintenant des performances acceptables, sans qu'il y ait altération de leur santé physique ou de leur bien-être. Du fait des modes d'élevage, les ruminants sont davantage soumis aux variations saisonnières du climat et doivent souvent s'adapter aux fluctuations des ressources alimentaires, alors que les porcs et les volailles sont généralement élevés dans des bâtiments où l'éleveur contrôle l'ambiance et les apports alimentaires. Les systèmes d'élevage des ruminants sont aussi beaucoup plus diversifiés et l'élevage de porcs est souvent perçu comme plus homogène, le même système dominant se retrouvant dans la plupart des pays du monde. De ce fait, la description explicite d'interactions génotype $x$ environnement, qui classiquement permettent d'évaluer la robustesse des animaux ou leur plasticité, est plus difficile à réaliser. La réalité est cependant plus complexe qu'il n'y paraît. En effet, parrallèlement au système dominant, plusieurs systèmes peuvent coexister dans un même pays, avec des objectifs de production et des modalités d'élevage parfois très différents. D'autre part, le système d'élevage dominant est «reproduit» tel quel dans différentes zones du monde alors que les conditions de climat, de disponibilité et de nature des aliments ou encore l'expertise des éleveurs peuvent être très différentes.
En France, le système dominant de production porcine représente plus de $95 \%$ de la production nationale. Il se caractérise par l'élevage des animaux en environnement fermé, généralement sur caillebotis, l'alimentation se faisant essentiellement à base d'aliments complets constitués de céréales, de tourteaux et de coproduits de l'alimentation humaine. Dans les élevages de production, les truies sont très majoritairement issues de croisements, le plus souvent un croisement Landrace $x$ Large White ou contenant des origines asiatiques. Les verrats sont issus de lignées synthétiques, des races pures Piétrain, Large White et Duroc, ou de croisements entre ces races. La relative homogénéité des systèmes d'élevage porcin à l'échelle internationale est favorisée par la mondialisation de la production qui a accéléré les échanges d'animaux et des systèmes d'élevage entre pays. Ainsi, au cours du 20 ème siècle, trois races ont connu une très large dissémination (Large White, Duroc et Landrace) et se retrouvent présentes sur tous les continents. Toutefois, comparativement au marché de la génétique avicole qui est particulièrement concentré, la génétique porcine apparaît plus diversifiée. On identifie un nombre limité d'organisations de sélection à vocation multinationale, mais elles sont loin d'avoir une position dominante (10-15\% du marché mondial) comme c'est le cas en volailles. De plus, en raison d'échanges internationaux d'animaux ou de semences rendus de plus en plus difficiles pour des raisons sanitaires, des spécificités «régionales» apparaissent au sein des races ou des lignées avec des objectifs de sélection influencés par le contexte local, en particulier climatique.

La relative homogénéité des modes de production porcine rencontrés dans le monde en termes de logement ou de choix génétiques, cache aussi une assez grande hétérogénéité de pratiques et de conduites d'élevage. Selon les pays, l'élevage est organisé en ateliers spécialisés par phases (reproduction, sevrage, engraissement) ou, comme c'est le plus souvent le cas en France, en ateliers assurant à la fois la production des porcelets et leur engraissement. La taille des élevages est également très variable. En France, elle va de quelques dizaines de truies pour les élevages les plus petits à quelques centaines pour les élevages spécialisés (Ilari et al 2004, 2007), alors que dans certains pays (Brésil et EtatsUnis par exemple) on peut trouver des élevages de plusieurs milliers, voire plusieurs dizaines de milliers, de truies. La conduite des truies diffère également entre les élevages d'un même pays, aussi bien en termes de durée de lactation qu'en nombre de bandes de reproductrices, influençant ainsi la répartition des événements (insémination, mise bas, sevrage...) au cours du temps et l'organisation du travail (Martel et al 2008). On retrouve cette variation entre élevages pour les stratégies de renouvellement-réforme des truies et les pratiques de surveillance des mises bas, de 
détection d'œstrus et d'insémination (Martel et al 2009). On peut alors s'interroger sur la capacité d'adaptation des animaux à cette diversité des conduites et des pratiques rencontrées dans les élevages conventionnels. Cette question se pose de façon encore plus marquée pour les élevages «alternatifs» dans lesquels les animaux sont plus soumis aux variations de l'environnement, notamment lorsqu'ils sont en plein air, ou présentent un risque accru de déficit nutritionnel, tout au moins à certaines périodes du cycle d'élevage, du fait de contraintes spécifiques sur les ingrédients autorisés.

\section{1 / Potentiel et performan- ces des truies reproduc- trices}

\section{1 / Évolution des performances des truies dans les élevages}

$\mathrm{Au}$ cours des 25 dernières années, la productivité numérique des truies a beaucoup augmenté (tableau 1). Les nombres de porcelets nés totaux et sevrés par portée se sont ainsi largement accrus, de respectivement 30 et $29 \%$ sur cette période. L'amélioration de la prolificité a eu lieu principalement durant les 15 dernières années et elle est le résultat de la sélection de lignées hyperprolifiques (Legault et Gruand 1976), de l'utilisation de lignées sino-européennes (Legault 1998) et de la mise en place d'une sélection intense pour la taille de portée dans les lignées maternelles depuis le début des années 1990 (Bidanel et Ducos 1994). Mais cette sélection s'est aussi accompagnée d'un accroissement du nombre de porcelets mort-nés et du taux de pertes des porce-

Figure 1. Évolution de la taille de la portée à la naissance et de la mortalité totale des porcelets (mort-nés + morts en cours d'allaitement) (d'après IFIP, base de données GTTT).

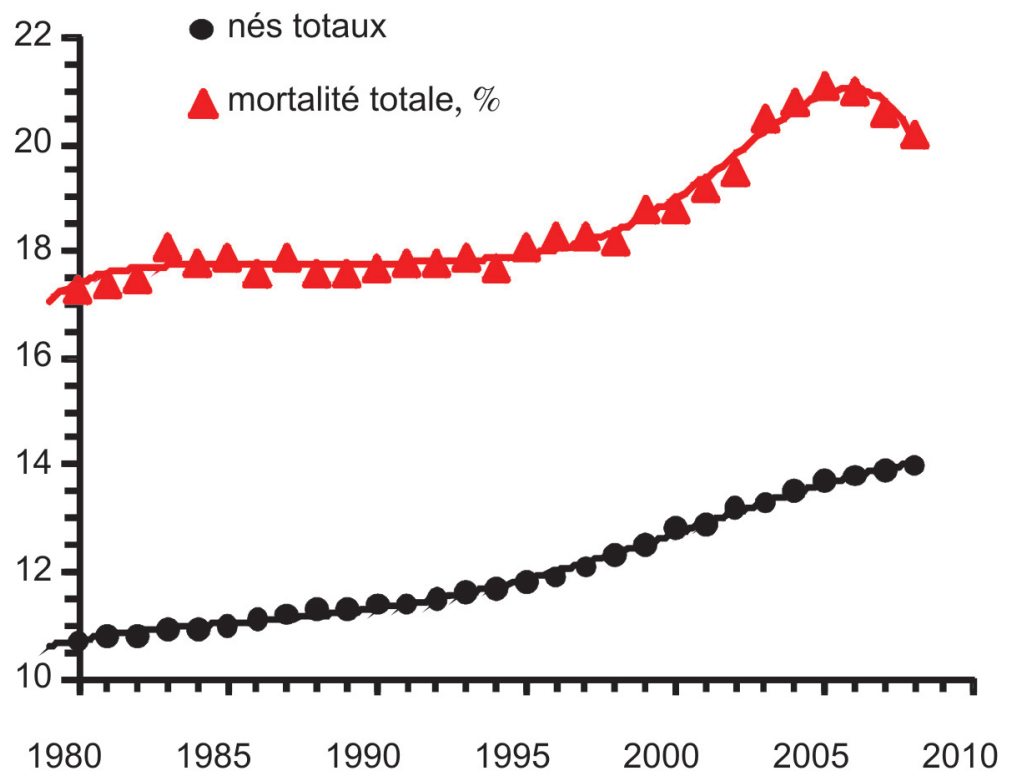

Année

lets pendant l'allaitement (figure 1). Cette augmentation phénotypique de la mortalité précoce a été confirmée dans le cadre d'un dispositif d'estimation des évolutions génétiques intervenues en France entre 1977 et 1998 (Canario et al 2007a). Pour limiter l'accroissement de la mortinatalité, le critère de sélection pour la prolificité a été modifié en 2002, en remplaçant le nombre de nés totaux par le nombre de nés vivants. En accord avec les résultats de Canario et al (2006a), une réduction des pertes à la mise bas est déjà observée dans les populations commerciales (figure 1). Parrallèlement à l'accroissement de la prolificité, on observe une accélération

Tableau 1. Évolution des performances de reproduction des truies entre 1980 et 2008 (d'après IFIP, base de données de gestion technique des troupeaux de truie, GTTT).

\begin{tabular}{|l|c|c|c|}
\hline & 1980 & 1995 & $\mathbf{2 0 0 8}$ \\
\hline Taille de portée & 10,8 & 11,8 & 14,0 \\
$\quad$ Nés totaux & 0,5 & 0,7 & 1,1 \\
Mort-nés & 8,9 & 9,7 & 11,2 \\
Sevrés & 13,6 & 13,0 & 13,7 \\
Pertes sur nés vivants, \% & 17,6 & 18,1 & 20,2 \\
$\quad$ Pertes sur nés totaux, \% & & & \\
Rythme de reproduction & 30 & 27 & 24 \\
$\quad$ Durée de lactation, j & 18,0 & 10,6 & 8,8 \\
Intervalle sevrage-fécondation, j & 2,25 & 2,39 & 2,46 \\
$\quad$ Portées par truie et par an & & & \\
Productivité numérique & 20,0 & 23,1 & 27,5 \\
Porcelets sevrés par truie par an & & & \\
Carrière & 4,3 & 4,7 & 5,1 \\
$\quad$ Portées sevrées & 38 & 45 & 57 \\
$\quad$ Porcelets sevrés & 33 & 33 & 34 \\
\hline Age à la réforme, mois &
\end{tabular}

du rythme de reproduction entre 1980 et 2008 (tableau 1). Ceci s'explique à la fois par une réduction de l'intervalle sevrage-fécondation, qui a diminué de 10 jours sur cette période, et de la durée de la lactation, qui est passée progressivement de 5 à 4, voire 3 semaines. La réduction de la durée de l'intervalle sevrage-fécondation résulte d'un raccourcissement de l'intervalle sevrageœstrus et surtout de l'amélioration du taux de fécondation à la première insémination, qui atteignait $90 \%$ en moyenne en 2008. Un effet d'hétérosis étant généralement observé sur les paramètres de fertilité, la généralisation de l'utilisation de truies croisées dans les années 1980 explique, au moins en partie, cette réduction de l'intervalle sevrage-fécondation. Depuis 1980, l'accélération du rythme de reproduction a permis d'accroître d'environ $10 \%$ le nombre de portées sevrées par truie et par an. Au final, l'accroissement combiné du nombre de porcelets sevrés par portée et du nombre de portées sevrées par an a conduit à une amélioration régulière de la productivité numérique des truies, qui est passée de 20 à près de 28 porcelets sevrés par an entre 1980 et 2008, soit une augmentation de $40 \%$. En parallèle, le nombre total de porcelets morts par truie et par an est passé d'environ 4 à $7 \mathrm{du}$ fait de l'accroissement du nombre de portées et de la mortalité des porcelets. La robustesse des animaux semble donc avoir légèrement diminué sur ce point.

L'amélioration de la productivité numérique des truies ne s'est pas faite au détriment de leur capacité de 
Tableau 2. Comparaison des performances de reproduction des truies en élevages conventionnels (bâtiment), plein air et biologiques (d'après IFIP-base de données GTTT et Prunier et Lebret 2009).

\begin{tabular}{|l|c|c|c|c|}
\hline & Élevage en & Élevage & \multicolumn{2}{|c|}{ Élevages biologiques } \\
\cline { 4 - 5 } & bâtiment & plein air & Moyens & Performants $^{1}$ \\
\hline Taille de portée & & & & \\
$\quad$ Nés totaux & 13,9 & 13,2 & 12,5 & 13,6 \\
Nés vivant & 12,8 & 12,2 & 11,4 & 12,6 \\
$\quad$ Sevrés & 11,0 & 10,0 & 9,0 & 9,9 \\
Pertes & & & & \\
$\quad$ sur nés vivants, \% & 20,6 & 24,2 & 31,0 & 27,2 \\
$\quad$ sur nés totaux,\% & 14,0 & 18,1 & 21,1 & 21,4 \\
Porcelets sevrés/truie/an & 27,5 & 24,0 & 19,4 & 21,4 \\
\hline
\end{tabular}

${ }^{1}$ les $50 \%$ des élevages les plus performants.

reproduction puisque, comme on a pu le voir ci-dessus, les paramètres «retour en œstrus après sevrage» et «taux de fécondation 1ère IA» se sont améliorés. Un autre aspect important à considérer est la longévité des animaux. Si, pour l'évaluer, on se base sur l'âge des truies à la réforme dans les élevages de production, on constate que cette longévité a peu évolué au cours des 25 dernières années (tableau 1). Elle s'est par contre un peu améliorée si l'on considère le nombre moyen de portées sevrées par truie avant la réforme qui est passé de 4,3 à 5,1 (tableau 1).

Les mêmes types génétiques de truies qu'en élevage conventionnel sont généralement utilisés en élevage plein air et en élevage biologique, ces derniers étant aussi en majorité conduits en plein air (Prunier et Lebret 2009). La comparaison des performances de ces différents types d'élevage pour un type génétique donné et sur une même période de temps nous donne donc des indications sur la capacité des truies à exprimer leurs performances dans des milieux différents (tableau 2). Comparativement

Figure 2. Comparaison du nombre de porcelets nés vivants par portée dans différentes races (sélectionnées $\square$ ou locales $\square$ ) et dans différents systèmes d'élevage (ם).

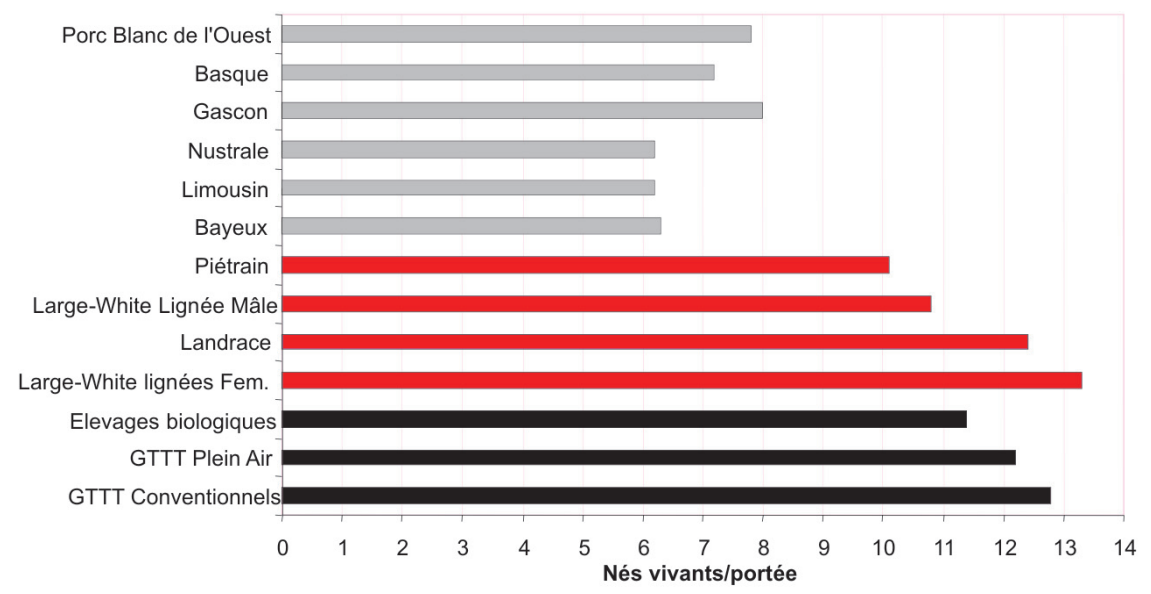

élevés dans les élevages biologiques. Selon cet auteur, cette mortalité accrue serait liée à l'allongement de la durée de la lactation et surtout au regroupement des truies après deux semaines de lactation, imposé en Suède dans les élevages biologiques. Par contre, les causes de mortalité ne varient pas selon le système. Ces résultats tendent donc à montrer que les moindres performances de reproduction observées dans les élevages biologiques, souvent conduits en plein air, sont principalement liées à des conditions de milieu, en particulier thermique, moins favorables pour les porcelets. Toutefois, la plus grande variabilité des performances observée entre les élevages dans les systèmes plein air et biologiques semble indiquer également que les pratiques d'élevage n'y sont pas toujours optimisées.

Les races porcines locales sont peu utilisées et le plus souvent dans des filières spécifiques à haute valeur ajoutée $(0,2 \%$ des truies, Lenoir et Mercat 2008). Comme l'indique la figure 2 , leurs performances de reproduction $(6$ à 8 porcelets nés vivants) sont nettement inférieures à celles des races sélectionnées. Par ailleurs, ces races, dans leurs conditions habituelles d'élevage, présentent généralement des taux de mortalité des porcelets élevés pendant la lactation, ce qui conduit à des tailles de portée très faibles au sevrage.

\section{2 / Adaptation des truies à l'accroissement de la taille de la portée}

Pour la truie, une première conséquence de l'accroissement de la prolificité est l'augmentation de la prise de poids pendant la gestation. Cette prise de poids correspond à la fois au gain de poids maternel (fin de croissance de la truie et/ou reconstitution des réserves corporelles) et au développement de la portée et de la mamelle (Noblet et al 1985). L'augmentation de la prolificité se traduit par un accroissement 
Tableau 3. Influence de la taille de la portée sur les besoins nutritionnels de la truie en première gestation (calculé d'après InraPorc).

\begin{tabular}{|l|c|c|c|}
\hline \multicolumn{1}{|c|}{ Nombre de foetus } & 11 & 14 & 17 \\
\hline Aliment, kg/j & $2,66(100)^{1}$ & $2,71(102)$ & $2,77(104)$ \\
Énergie, MJ EM/j & $33,7(100)$ & $34,4(102)$ & $35,0(104)$ \\
Lysine digestible $^{2}, \mathrm{~g} / \mathrm{kg}$ & $4,91(100)$ & $5,27(107)$ & $5,72(116)$ \\
Phosphore digestible $^{2}, \mathrm{~g} / \mathrm{kg}$ & $2,54(100)$ & $2,76(109)$ & $2,98(117)$ \\
\hline
\end{tabular}

1 Entre parenthèses : valeurs exprimées en base 100.

2 Pendant le dernier tiers de la gestation.

relativement limité des besoins énergétiques (tableau 3 ) et, à cette période, l'appétit des truies est toujours suffisant pour leur permettre de couvrir ces besoins. En revanche, les besoins relatifs en acides aminés et en minéraux augmentent de façon plus marquée, en particulier en fin de gestation, lorsque la croissance des fœetus s'accélère. Ceci rend les truies hyperprolifiques plus exigeantes en termes de qualité des aliments et donc plus susceptibles de présenter des carences si les apports sont insuffisants, comme cela peut être le cas dans certains systèmes d'élevage biologique, compte tenu de contraintes spécifiques sur l'origine des aliments. Au total, la prise de poids pendant la gestation peut atteindre 50 à $90 \mathrm{~kg}$, ce qui représente 40 à $60 \%$ du poids à l'insémination pour les truies en première gestation. Bien que peu de données existent sur ce point, on peut s'interroger sur les risques pour les aplombs de cette très forte prise de poids chez des femelles encore en croissance. Les problèmes d'aplombs expliquent 9 à $14 \%$ des réformes chez les cochettes et les truies primipares, selon Lucia et al (2000) et Englom et al (2007), entraînant à la fois des pertes économiques et des problèmes de bien-être pour les animaux. Dans l'étude de Heinonen et al (2006), environ $10 \%$ des jeunes truies et $7 \%$ des truies multipares présentent des boiteries, et dans la moitié des cas, en raison d'ostéochondrose ou d'arthrite. La survenue de ces problèmes peut être exacerbée par certains types de logement en relation, notamment, avec le type de sol (caillebotis). Dans le cas des truies gravides élevées en groupes, comme cela sera obligatoire à partir de 2013, il est important de limiter la compétition sociale en offrant aux animaux une surface au sol suffisante et un système

Tableau 4. Influence de la saison et de la race sur les performances des truies allaitantes en milieu tropical (Gourdine et al 2006).

\begin{tabular}{|l|c|c|c|c|}
\hline \multicolumn{1}{|c|}{ Race } & \multicolumn{2}{c|}{ Créole } & \multicolumn{2}{c|}{ Large White } \\
\hline Température & Fraîche & Chaude & Fraîche & Chaude \\
\hline Appétit des truies, $\mathrm{kg} / \mathrm{j}^{1}$ & $3,44(100)$ & $2,97(86)$ & $4,70(100)$ & $3,82(81)$ \\
GMQ des porcelets, $\mathrm{g} / \mathrm{j}^{1}$ & $199(100)$ & $191(96)$ & $224(100)$ & $206(92)$ \\
\hline
\end{tabular}

1 Entre parenthèses : pourcentage de la valeur mesurée en saison fraîche, intra race. tion, entraînant une élévation de la température corporelle et du rythme respiratoire et vraisemblablement une situation d'inconfort pour l'animal. En milieu tropical, Gourdine et al (2006) ont mis en évidence une sensibilité moindre des truies Créoles à la saison chaude comparativement aux truies Large White, leur appétit et la croissance de leurs porcelets étant moins affectés par la saison (tableau 4). De plus, la température interne était davantage augmentée en saison chaude chez les truies Large White que chez les truies Créoles, soulignant une difficulté plus grande des premières à s'adapter à des températures élevées. Les truies Créoles sont donc plus robustes mais leurs performances sont moins élevées quelle que soit la saison.

Les effets négatifs des déficits nutritionnels sur les performances de reproduction ont été décrits dans différentes synthèses bibliographiques (Prunier et Quesnel 2000, Quesnel 2005). Dans la plupart des études conduites ces 20 dernières années, un déficit nutritionnel sévère retarde le retour en œstrus après le sevrage et diminue la fertilité et la taille de la portée suivante, ces effets étant surtout marqués chez les truies primipares. Toutefois, dans des études plus récentes, le retour en œstrus semble être moins affecté, la mortalité embryonnaire étant cependant toujours accrue (Quesnel 2009). Ceci semble en accord avec les observations de terrain qui ne mettent pas en évidence d'augmentation du délai de retour en œstrus après sevrage chez les truies hyperprolifiques (tableau 1), malgré un accroissement vraisemblable du déficit nutritionnel. $\mathrm{La}$ réduction de la survie embryonnaire a finalement peu d'impact en pratique puisque la prolificité moyenne est très élevée dans les élevages.

Dans les conditions conventionnelles de production, l'accroissement de la prolificité des truies et de leur production laitière ne semble donc pas avoir eu d'effets négatifs sur la fonction de reproduction, hormis un accroissement de la mortalité des porcelets. Toutefois, lorsque l'environnement climatique devient moins favorable, les performances des truies se dégradent, ce qui souligne des difficultés d'adaptation. Certaines races locales semblent mieux résister aux variations climatiques mais leurs performances de reproduction sont relativement faibles.

\section{3 / Adaptation des porcelets à l'accroissement de la taille de la portée}

L'accroissement de la taille de la portée peut entraîner une compétition accrue pour les nutriments et l'espace, 
aussi bien pendant la vie embryonnaire et fotale qu'après la naissance. Cette compétition accrue est susceptible d'entraîner la mortalité de certains porcelets et/ou d'accroître la variabilité de leur développement. L'accroissement de la prolificité semble favoriser des durées de parturition un peu plus longues. En effet, Canario et al (2006a) ont montré que même si le rythme des naissances est accéléré dans les portées plus nombreuses, la durée totale de la mise bas augmente avec le nombre de porcelets nés.

a) Compétition entre les foetus pour l'espace utérin et les nutriments

Le développement et la survie des embryons et des fœtus in utero ont été beaucoup étudiés dans les années 80, c'est-à-dire chez des truies moins prolifiques qu'aujourd'hui. Dans ces études, la mortalité avait lieu en majorité avant 30 jours de gestation (20 à $40 \%$ des embryons) et la mortalité tardive était faible (5 à 10\% des foetus) (Ashworth et Pickard 1998). La mortalité embryonnaire ne s'est pas accrue chez les truies hyperprolifiques selon Vonnahme et al (2002) et Town et al (2005). Une mortalité embryonnaire faible (15\%) est même notée chez des truies hyperprolifiques nullipares aboutissant en moyenne à 17 embryons vivants au 27 ème jour de gestation (Quesnel et al 2009). Ceci conduit, chez les truies hyperprolifiques, à une surpopulation des cornes utérines qui entraîne une seconde vague de mortalité entre 30 et 55 jours de gestation (Vonnahme et al 2002, Town et al 2005). Cette deuxième vague de mortalité intervient lorsque le placenta est en plein développement, ce qui peut conduire à une réduction du développement des fœtus restants, comme cela a été montré par Père et al (1997) et plus récemment par Town et al (2005) et Tse et al (2008).

L'accroissement du nombre de fœtus s'accompagne d'une augmentation du flux total de sang vers l'utérus mais aussi d'une réduction du flux par fœetus et, par conséquent, de l'apport de nutriments (Père et Etienne 2000), ce qui doit conduire logiquement à une réduction du poids de naissance des porcelets. Ceci est confirmé par l'analyse de l'influence de la taille de la portée sur le poids des porcelets à la naissance (figure 3, Quesnel et al 2008), indiquant qu'il y a plus de porcelets de petits poids $(<1,0 \mathrm{~kg})$ et moins de porcelets lourds $(>1,8 \mathrm{~kg})$ dans les portées de plus grande taille. Néanmoins, Canario et al (2007a) ont estimé qu'à taille de portée égale, les porcelets issus des truies actuelles sont plus lourds à la naissance que les porcelets produits par des truies de 1977. En revanche, leur maturité à la naissance s'est dégradée (Canario et al 2007b). Les porcelets présentent en effet une composition corporelle à la naissance moins favorable, avec moins de matière sèche et d'énergie dans leur carcasse, un poids du foie inférieur et des réserves plus limitées de glycogène hépatique. La concentration plasmatique d'IGF-I a également diminué (Canario et al 2007a et b). Toutefois, malgré une teneur en protéines corporelles plus faible à la naissance, les porcelets actuels présentent un potentiel de croissance supérieur, qui s'exprime après la naissance (Canario 2006, Canario et al 2007b). Ces résultats confirment ceux obtenus par Herpin et al (1993) qui suggéraient, sur la base de comparaisons entre races, que la sélection sur la croissance musculaire influence la maturité physiologique et la composition corporelle des nouveaunés, les porcelets issus des lignées à croissance rapide étant, à la naissance, à la fois plus lourds et moins matures.

b) Consommation de colostrum et de lait par les porcelets

Bien que la production de lait n'ait pas été intégrée dans les critères de sélection, on constate qu'elle s'est fortement accrue avec l'évolution de la prolificité (synthèse de Étienne et al 2000, Canario et al 2006b). Ceci est en partie lié à l'influence connue de la taille de la portée sur la production de lait, mais cet effet n'explique pas tout puisque Etienne et al (2000) montrent que la quantité de lait disponible par porcelet a également augmenté, en accord avec l'accroissement du poids des porcelets au sevrage. $\mathrm{Au}$ final, ces auteurs estiment que la

Figure 3. Influence de la taille de la portée sur la répartition des poids des porcelets à la naissance (Quesnel et al 2008).

\section{$\%$ de nés totaux}

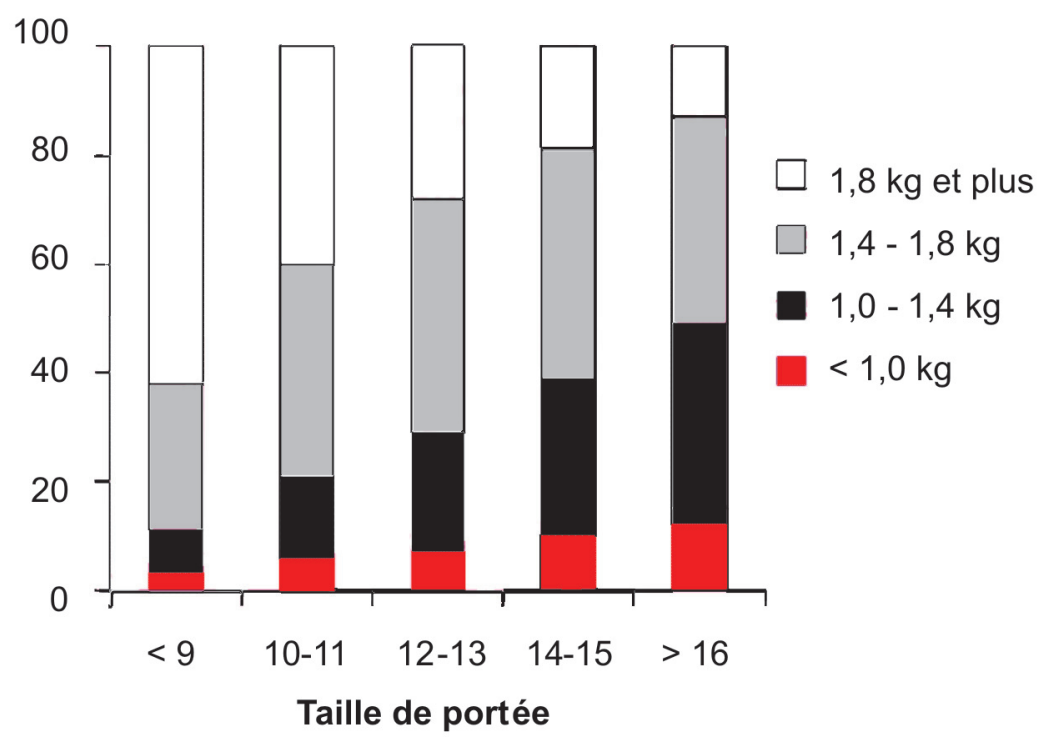

production de lait a doublé au cours des 30 dernières années, mais les mécanismes impliqués, en dehors de l'effet de l'accroissement de la taille de la portée, ne sont pas clairement identifiés. Toutefois, il est intéressant de noter que, suite à la sélection, le nombre de tétines fonctionnelles s'est accru de près d'une unité depuis 10 ans (Tribout et al 2003, Canario et al 2006b).

La production de colostrum des truies semble par contre avoir peu évolué (Canario 2006). Devillers et al (2007) montrent que la quantité de colostrum produit par la truie dépend peu de la taille de la portée et que, par conséquent, la quantité disponible par porcelet diminue significativement dans les portées de plus grande taille. Par ailleurs, ces auteurs soulignent l'existence d'une forte variabilité entre truies pour la production de colostrum. donc accompagné d'une fréquence accrue de porcelets de petits poids à la naissance et d'une consommation réduite de colostrum alors que la quantité de lait disponible par porcelet semble avoir augmenté. On peut s'interroger sur les conséquences de cette évolution sur la survie des porcelets et leur bien-être. En effet, la consommation de colostrum en quantité suffisante est nécessaire pour couvrir les besoins énergétiques des porcelets nouveau-nés et leur assurer une protection immunitaire (synthèse de Le Dividich et al 2005). Les porcelets légers, moins vigoureux, consomment moins de colostrum (Devillers et al 2007), ce qui réduit leurs chances de survie pendant les premiers jours de
L'accroissement de la prolificité s'est 
Tableau 5. Évolution des performances de croissance des porcelets en post-sevrage et des porcs à l'engrais entre 1988 et 2008 (d'après IFIP, base de données de gestion technico-économique (GTE), élevages naisseurs-engraisseurs).

\begin{tabular}{|c|c|c|c|c|}
\hline & \multirow{2}{*}{1988} & \multirow{2}{*}{1998} & \multicolumn{2}{|c|}{2008} \\
\hline & & & Moyenne & CV\% ${ }^{1}$ \\
\hline \multicolumn{5}{|l|}{ GMQ, $\mathrm{g} / \mathrm{i}$} \\
\hline post-sevrage & 426 & 473 & 471 & 9,1 \\
\hline engraissement & 666 & 767 & 777 & 7,1 \\
\hline \multicolumn{5}{|l|}{$\mathrm{IC}, \mathrm{kg}$ aliment $/ \mathrm{kg}$ gain } \\
\hline post-sevrage & 2,02 & 1,76 & 1,70 & 13,4 \\
\hline engraissement & 3,19 & 2,93 & 2,89 & 6,2 \\
\hline Age à l'abattage, j & 195 & 183 & 186 & 5,9 \\
\hline Poids d'abattage, kg & 103 & 111 & 116 & 2,7 \\
\hline Taux de viande maigre ${ }^{2}$ & 55,5 & 58,6 & 60,2 & 1,3 \\
\hline
\end{tabular}

$1 \mathrm{CV}$ : coefficient de variation entre élevages.

2 Exprimé en équivalent TMP (Taux de Muscle des Pièces, kg/100 kg de carcasse).

lactation. Le taux de mortalité des porcelets pesant moins d'un kg à la naissance est ainsi de $25 \%$ alors qu'il est de seulement $5 \%$ pour les porcelets de plus de 1,6 kg (Quiniou et al 2002). De plus, la compétition pour les tétines est exacerbée dans les portées de grande taille, ce qui peut accroître les risques de lésions à la fois pour les porcelets et pour la truie (Norring et al 2006).

En résumé, l'accroissement de la taille de la portée fragilise les porcelets à la naissance et pendant la lactation. Cela se traduit dans les élevages par une légère dégradation du taux de survie des porcelets parallèlement à l'accroissement de la taille de la portée à la naissance (cf. supra).

c) Implications de l'accroissement de la prolificité en termes de conduite d'élevage

La conduite en bande des truies, en favorisant le regroupement des mises bas, facilite les adoptions et permet ainsi d'uniformiser les tailles de portée. Dans cette situation, certains éleveurs font parfois appel à des truies nourrices, qui sont généralement des truies d'une bande précédente dont la portée a été sevrée un peu plus tôt (Boulot et al 2008). De nouvelles pratiques de surveillance des mises bas et de soins aux porcelets se sont également développées (Martel et al 2009). Par exemple, des compléments énergétiques ou du colostrum congelé collecté sur d'autres truies peuvent être apportés pour pallier le déficit en colostrum des porcelets légers.

Ces pratiques semblent efficaces pour améliorer la survie des porcelets, mais elles demandent du temps supplémentaire et une excellente technicité de la part des éleveurs. De plus, elles sont difficilement applicables dans certains systèmes d'élevage. Ainsi, lorsque les truies sont libres pendant la mise bas, surtout si elles sont en plein air, il est beaucoup plus difficile de mettre ces pratiques en œuvre, compte tenu du comportement parfois agressif des mères qui «protègent» leurs porcelets, les interventions humaines pouvant même parfois conduire à un accroissement de la mortalité (Berger et al 1997). Par ailleurs, dans certains systèmes d'élevage n'autorisant pas le recours à des hormones, les mises bas sont moins bien synchronisées et/ou les bandes sont de plus petite taille, ce qui rend les adoptions plus difficiles.

\section{2 / Potentiel et performan- ces des animaux sevrés et des porcs à l'engraissement}

\section{1 / Évolution des performances dans les élevages}

L'évolution au cours des 20 dernières années des performances de croissance dans les élevages français est présentée dans le tableau 5. En 20 ans, la vitesse de croissance s'est améliorée de respectivement 14 et $17 \%$ pendant les périodes de post-sevrage et d'engraissement. Cette amélioration de la vitesse de croissance s'est accompagnée d'une réduction de l'indice de consommation de respectivement 16 et $10 \%$ pour les deux stades de croissance. Au cours de cette même période, le taux de viande maigre des carcasses s'est accru de près de 5 points $(+9 \%)$ malgré un accroisse- ment du poids d'abattage de $13 \mathrm{~kg}$ $(+13 \%)$. Cette évolution des performances est en grande partie le résultat de la sélection d'animaux à croissance plus rapide, qui déposent plus de protéines et moins de gras, ce qui réduit le coût énergétique du gain de poids et donc l'indice de consommation. Une part importante de l'accroissement du potentiel de croissance a été valorisée pour produire des carcasses à la fois plus lourdes et plus maigres, l'âge à l'abattage étant réduit de seulement 9 jours en moyenne. Il existe par ailleurs une variabilité importante des performances entre les élevages, en particulier pour la vitesse de croissance et surtout l'indice de consommation.

La comparaison des performances mesurées dans les élevages à celles obtenues dans le cadre des tests de produits terminaux, avec des origines génétiques similaires, permet d'avoir une idée de la façon dont les animaux réalisent leur potentiel génétique en conditions de production commerciale (tableau 6). Il en ressort que le potentiel génétique ne s'exprime pas complètement en situation de terrain. La vitesse de croissance est ainsi supérieure de $21 \%$ et l'indice de consommation inférieur de $8 \%$ dans les conditions privilégiées des tests de contrôle de terminaux. En revanche, aucune différence n'est observée pour la composition des carcasses. Il est vraisemblable que les conditions commerciales d'élevage, et en particulier le rationnement alimentaire pratiqué dans beaucoup d'élevages pendant la phase d'engraissement, ou généré par la compétition entre les animaux, expliquent en grande partie l'écart de vitesse de croissance. En comparant différents types de logement, Dourmad et al (2009) ont aussi observé des effets importants sur les performances des animaux (tableau 7). Le logement conventionnel, avec $0,65 \mathrm{~m}^{2} /$ porc sur caillebotis intégral, conduit à des performances moindres de croissance qu'un logement sur litière associé à une courette extérieure $\left(2,5 \mathrm{~m}^{2} /\right.$ porc $)$.

L'évolution, au cours des 20 dernières années, des taux de mortalité des porcelets en post-sevrage et en engraissement montre des changements contrastés selon les périodes considérées

Tableau 6. Comparaison des performances obtenues en engraissement dans les élevages suivis en GTE (2008) et dans les tests de terminaux réalisés en station publique (2008).

\begin{tabular}{|l|c|c|c|}
\hline & Élevages & $\begin{array}{c}\text { Test de } \\
\text { terminaux }\end{array}$ & $\begin{array}{c}\text { Différence } \\
(\%)\end{array}$ \\
\hline GMQ, g/j & 777 & 940 & +21 \\
IC, kg aliment/kg gain & 2,89 & 2,65 & -8 \\
TMP, \% & 60,2 & 60,4 & \\
\hline
\end{tabular}


Tableau 7. Influence du mode de logement ${ }^{1}$ sur les performances de croissance de porcs nourris ad libitum (d'après Dourmad et al 2009).

\begin{tabular}{|l|c|c|c|}
\hline \multicolumn{1}{|c|}{ Logement } & Caillebotis & $\begin{array}{c}\text { Litière et } \\
\text { courette }\end{array}$ & $\begin{array}{c}\text { Différence } \\
\mathbf{( \% )}\end{array}$ \\
\hline Aliment, kg/j & 2,71 & 2,92 & $+7,7$ \\
GMQ, g/j & 950 & 1027 & +8 \\
IC, kg aliment/kg gain & 2,81 & 2,80 & \\
TMP, \% & 60,7 & 59,1 & $-2,6$ \\
\hline
\end{tabular}

${ }^{1}$ Caillebotis : 0,65 m²/porc; litière + courette : 1,2 $\mathrm{m}^{2} /$ porc pour litière $+1,1 \mathrm{~m}^{2} /$ porc pour courette extérieure.

(figure 4). Jusqu'à 1995, on note une tendance à la réduction de la mortalité des porcelets en post-sevrage alors qu'au contraire la mortalité des porcs en engraissement tend à s'accroître, la mortalité totale restant relativement stable, autour de 5,5\%. Entre 1995 et 2004, la mortalité augmente très significativement chez les porcs à l'engrais alors qu'elle évolue peu en post-sevrage, la mortalité totale atteignant $8 \%$ en 2004. $\mathrm{Au}$ cours des 5 dernières années, la mortalité totale diminue fortement et, en 2009, elle est voisine de $5 \%$. L'augmentation de la mortalité des animaux jusqu'en 2004 peut être interprétée comme la marque d'un accroissement de la sensibilité des animaux à des problèmes pathologiques, en liaison notamment avec l'expansion du syndrome de la maladie d'amaigrissement du porcelet (MAP) (Rose et al 2003). La nette amélioration qui suit illustre une certaine «élasticité» des animaux ou des élevages dans la mesure où, après une période de dégradation, les performances retrouvent leur niveau initial.

Figure 4. Évolution de la mortalité des porcs en post-sevrage et en engraissement ${ }^{1}$ (d'après IFIP, base de données GTE).

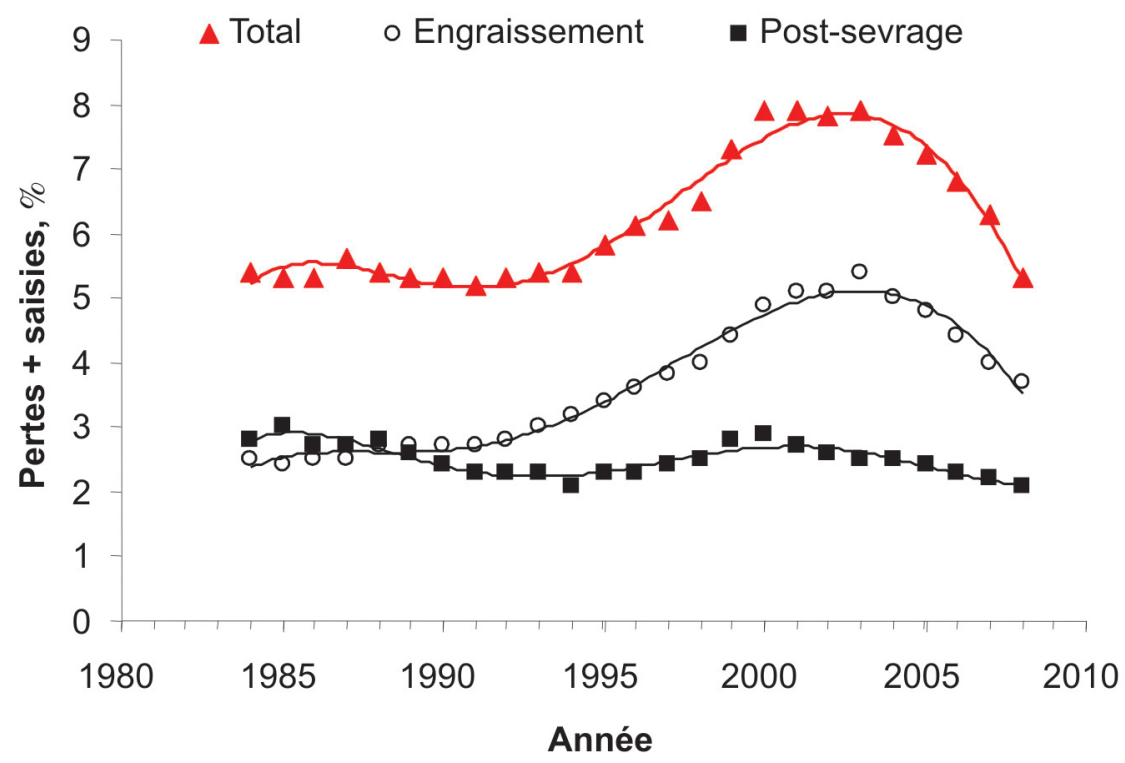

${ }^{1}$ Pour l'engraissement les pertes regroupent les mortalités et les saisies en abattoir. leurs conditions d'élevage au moment du sevrage.

\section{2 / Capacité à répondre à un challenge immunitaire}

Comme on l'a vu ci-dessus, les performances des porcs se sont largement améliorées en réponse à la sélection (Tribout et al 2004), avec un fort accroissement de la vitesse de croissance et de l'efficacité alimentaire. Cette sélection d'animaux plus productifs pourrait avoir entraîné une altération de leur capacité à faire face à des challenges immunitaires (Rauw et al 1998). D'après la théorie de l'allocation des ressources, les différentes fonctions biologiques d'un animal sont en compétition vis-à-vis des ressources nutritionnelles. Chez les animaux à fort potentiel, le tissu musculaire et la fonction de croissance seraient prioritaires sur d'autres fonctions telles que, par exemple, la fonction immunitaire, qui est pourtant forte consommatrice d'énergie et d'acides aminés lorsqu'elle est activée par une infection microbienne (Le Floc'h 2000). D'autre part, la sélection sur la croissance musculaire a corrélativement altéré la sécrétion d'hormones métaboliques qui ont aussi un rôle de contrôle immunitaire (Prunier et al 2010). Par exemple, les races maigres présentent souvent des concentrations plasmatiques de cortisol plus basses que les races grasses (Shaw et al 1995, Désautés et al 1997). Or, le cortisol joue un rôle important dans le contrôle du métabolisme et est une hormone fondamentale dans le contrôle de la réponse immunitaire (Merlot 2004). Même si de telles corrélations négatives entre croissance et immunité ont été mises en évidence chez les volailles (Rauw et al 1998), peu d'informations viennent étayer cette hypothèse chez le porc. Quelques observations sur des lignées sélectionnées de façon divergente pour le dépôt de tissu maigre ont montré des variations de la formule sanguine et des caractéristiques des lymphocytes circulants sans qu'il soit réellement possible d'en tirer des conclusions sur une éventuelle modification de leur capacité à réagir face à un pathogène (Clapperton et al 2005 et 2008). Récemment, Merlot et al (2010) ont comparé des porcs Basque ayant une croissance lente orientée vers un dépôt de tissu adipeux très élevé et des porcs Large White ayant une croissance rapide orientée vers un fort dépôt de tissu maigre, et ceci dans deux milieux d'élevage (conventionnel sur caillebotis intégral ou sur litière avec un accès une courette extérieure). Dans cette étude, l'interaction race $x$ logement tendait à être significative pour de nombreux paramètres immunitaires (immunoglobulines circulantes, formule sanguine, 
prolifération lymphocytaire), la concentration plasmatique en haptoglobine (marqueur de l'inflammation) et la sévérité des ulcères gastriques. Le plus souvent, l'interaction révélait que les porcs Large White étaient plus sensibles aux variations de mode de logement que les porcs Basque, suggérant une plus grande sensibilité de ces animaux aux effets du logement.

En termes d'amélioration génétique, la réponse immunitaire des animaux est classiquement évaluée selon deux axes considérés comme complémentaires : le premier vise à sélectionner des animaux résistants à des pathologies spécifiques, le second vise à les sélectionner pour une meilleure immunité générale. En production porcine, il existe relativement peu de pathologies majeures, contrairement par exemple à la situation des vaches laitières qui doivent faire face de façon régulière à des risques de mammites. En revanche, comme nous l'avons rapporté précédemment, il existe des périodes de stress dans la vie de l'animal qui le rendent particulièrement sensible aux pathogènes : c'est le cas, par exemple, de la période de postsevrage, durant laquelle les animaux sont sensibles à certaines populations d'Escherichia coli, entraînant l'apparition de diarrhées. Une variabilité génétique des résistances à des pathogènes spécifiques est souvent présente dans les populations de production (Wilkie et Mallard 2000) et il est donc théoriquement possible de sélectionner les animaux les plus résistants. Le risque est cependant grand de voir la capacité de résistance ainsi sélectionnée contournée par la population de pathogènes cibles, ou bien de voir le remplacement de cette population par un autre pathogène dont l'effet est similaire (Luther et al 2009, Nielsen et al 2009). On peut donc considérer que, sauf pathologie majeure, ce type de sélection spécifique a un coût élevé, peut affecter la variabilité génétique de la population et présente un gain à moyen terme trop incertain pour être mené de façon systématique.

De façon alternative, la réponse immunitaire globale présente aussi une variabilité génétique qui peut permettre d'améliorer significativement le niveau moyen de santé des animaux par la sélection (Wilkie et Mallard 1999). Une sélection divergente sur la réponse immunitaire, à l'aide d'un indice combinant des marqueurs de la réponse humorale (anticorps) et des marqueurs de la réponse cellulaire, a donné des résultats encourageants en termes de réponses globales (Crawley et al 2005), mais elle a aussi montré l'ampleur de la difficulté à établir un critère intégrant l'ensemble de la réponse immunitaire et qui soit mesurable en routine pour per- mettre la sélection. Dans ce sens, de premiers essais réalisés sur l'utilisation de marqueurs moléculaires de l'immunité à l'aide d'une puce à ADN ciblée sur la région du complexe majeur d'histocompatibilité (Flori et al 2006), ainsi que la détection de QTL associés aux caractères de l'immunité (Hu et Reecy 2007), suggèrent la possibilité d'utiliser des marqueurs génétiques afin de sélectionner pour une meilleure réponse immunitaire.

\section{3 / Sensibilité au stress et com- portements anormaux}

Un exemple bien connu de relation entre les performances et la sensibilité au stress chez le porc est l'existence chez certaines races, en particulier le Piétrain, du gène RYR $1^{\mathrm{T}}$ aussi connu sous le nom de gène de la sensibilité à l'halothane. La présence de cet allèle à l'état homozygote $n n$ ou hétérozygote $\mathrm{Nn}$ accroît le développement musculaire et réduit l'adiposité des animaux. Mais cet allèle présent à l'état homozygote $n n$ rend les animaux fortement sensibles au stress, ce qui accroît le risque de mortalité et est à l'origine de défauts majeurs de qualité de la viande. L'élimination de l'allèle $n$ des lignées maternelles a permis d'éviter la production d'animaux terminaux $n n$. Cependant, compte tenu de l'utilisation fréquente en France de verrats terminaux de race Piétrain, la production d'animaux $\mathrm{Nn}$ est courante. Selon certains auteurs, ces animaux seraient plus sensibles au stress que les animaux $N N$. Ils présentent par exemple, lors du chargement et du transport, une température rectale et un rythme respiratoire plus rapides (Yoshioka et al 2004). Même si ces effets sont sans commune mesure avec ceux observés chez les animaux $n n$ et si l'élevage de ces animaux ne pose pas de problèmes pratiques importants, on peut s'interroger sur les conséquences en termes de bien-être animal de la production d'animaux $N n$.

Les types génétiques modernes à croissance rapide semblent plus susceptibles de développer des comportements anormaux comme les morsures de queue. Une corrélation génétique positive a ainsi été établie entre la vitesse de croissance du tissu maigre et la fréquence des actes de morsures de queue, alors que cette corrélation était négative avec l'épaisseur de gras dorsal (Breuer et al 2005). De même, on note des différences entre les races ; les animaux Duroc mordent davantage leurs congénères que les animaux Landrace ou Large White (Breuer et al 2005). On note également que la restriction alimentaire induit des modifications comportementales, en particulier une compétition alimentaire accrue dont les conséquences semblent plus sévères chez les animaux maigres que chez les animaux gras (Botermans et Svendsen 2000). Cette restriction alimentaire est probablement génératrice d'un stress plus important chez les types génétiques maigres que chez les types génétiques gras, menant à des concentrations de cortisol élevées et à une fréquence accrue d'ulcères gastriques (Merlot et al 2010). Dans une population suédoise, Canario et al (2008, 2010) ont montré que les porcs qui présentent un potentiel génétique supérieur pour la croissance sont plus agressifs.

\section{4 / Faiblesse des aplombs}

Les problèmes d'aplombs sont à l'origine de douleurs pour les animaux et de baisse des performances et, dans les cas les plus graves, ils conduisent à la réforme ou à l'abattage prématuré. L'ostéochondrose est considérée comme la principale cause des problèmes d'aplombs chez le porc. Il s'agit d'une maladie non-infectieuse caractérisée par une mauvaise ossification du cartilage. Les signes cliniques, principalement des boiteries, apparaissent entre 4 et 18 mois (Dewey 1999). L'étiologie de l'ostéochondrose n'est pas clairement établie et elle est généralement considérée comme d'origine multifactorielle mais avec une forte influence des facteurs intrinsèques, notamment génétiques (Ytrehus et al 2007). En effet, la prévalence diffère selon les races et les lignées et l'héritabilité est comprise entre 0,1 et 0,5 dépendant des sites osseux (Prunier et al 2010).

Certains auteurs ont montré qu'une croissance rapide serait un facteur prédisposant au développement de cette affection et qu'il y aurait une corrélation génétique positive entre le pourcentage de tissu maigre et la fréquence de l'ostéochondrose (Busch et al 2007), mais ces résultats ne sont pas retrouvés dans toutes les études (Prunier et al 2010). De la même manière, des facteurs alimentaires ont été avancés pour expliquer les problèmes d'ostéochondrose (Nakano et al 1987) mais, dans la majorité des études, la modification de l'alimentation est sans effet sur la fréquence ou la sévérité des lésions.

Des facteurs génétiques multiples sont mis en évidence pour ces pathologies, à travers des quantifications directes des affections des articulations ou des os, ou des notations des défauts de pattes et de locomotion. Cependant, à ce jour, seules quatre études rapportent des QTL affectant les aplombs (Guo et al 2009, Uemoto et al 2009, pour les plus récentes), ceci en raison des difficultés à obtenir des mesures systématiques standardisées de ces affections. Il n'y a donc 
pas d'indicateurs moléculaires disponibles à l'heure actuelle pour ce type de défaut.

$\mathrm{Au}$ final, le lien entre croissance rapide ou fort développement musculaire et ostéochondrose est mal établi mais il est clair que les facteurs génétiques jouent un rôle important, si bien qu'une meilleure prise en compte de la qualité des aplombs dans les schémas de sélection devrait permettre d'améliorer la robustesse des animaux sur ce point.

\section{Conclusions et perspectives}

L'analyse de l'évolution des performances des élevages porcins au cours des trente dernières années indique une amélioration significative de la productivité numérique et de la production laitière des truies, sans qu'il y ait a priori de détérioration de leur longévité. Cette amélioration s'explique à la fois par l'obtention de progrès génétiques et par la sophistication des pratiques d'élevage, notamment de l'alimentation. Bien que les performances se soient améliorées, on note, toutefois, que la survie des animaux s'est légèrement dégradée, en particulier aux alentours de la naissance et pendant la phase d'allaitement. A présent, les objectifs de sélection génétique visent la production de truies plus «autonomes» pendant la mise bas, plus aptes à élever leurs porcelets et dont la venue en ostrus est rapide et expressive. De tels animaux, plus rustiques, correspondent à un réel besoin aussi bien pour les élevages conventionnels que pour les élevages plein air ou biologiques, avec probablement des adaptations à définir pour chaque type de système.

La question se pose toutefois sur la capacité des truies ou des porcelets à faire face aux nombreux changements biologiques qui sous-tendent l'amélioration des performances. Chez la truie, on a ainsi pu identifier la problématique des aplombs et celle de la difficulté accrue des animaux à couvrir leurs besoins nutritionnels. Chez les porcelets, la question de la compétition pour les nutriments entre les individus d'une même portée in utero et durant la lactation se pose de façon plus marquée dans les grandes portées.

Dans les élevages, les performances de croissance des porcs se sont améliorées de façon marquée. Toutefois, l'écart important observé entre les performances de terrain et le potentiel génétique semble indiquer des problèmes d'adaptation des animaux. La sensibilité des porcs aux maladies d'origine multifactorielle constitue sûrement aujourd'hui l'un des facteurs les plus limitants dans les élevages. On peut ainsi s'interroger sur l'incidence qu'a pu avoir la sélection sur la capacité des animaux à résister à des challenges immunitaires ou à des situations de stress.

La relative homogénéité des résultats zootechniques rapportés dans cette étude, qui intègre la variabilité des conditions d'élevage, est un des indicateurs d'une relativement bonne robustesse des porcs dans les conditions actuelles de production. Il a été montré que les problèmes dits «de robustesse» sont généralement maîtrisés par des évolutions combinées des pratiques d'élevage, d'alimentation, de sélection et éventuellement de médications. L'accès à une diversité de ressources génétiques et le maintien de la variabilité génétique dans les populations sélectionnées demeurent, dans ce contexte, une nécessité, de façon à pouvoir s'adapter rapidement à de nouvelles questions. Finalement, l'émergence des outils moléculaires constitue, pour les caractères difficiles à mesurer en routine, un espoir pour la mise en œuvre de stratégies de sélection dans les populations de production.

En termes de perspectives, les questions liées à la robustesse doivent être approchées à la lumière des évolutions attendues des pratiques d'élevage et des réglementations. Par exemple, la compétition grandissante entre ressources pour l'alimentation animale et ressources pour l'alimentation humaine ou la production énergétique pose la question de la capacité des types génétiques actuels à réaliser leurs potentiels de croissance avec des aliments moins riches en énergie ou en protéines, ou enrichis en fibres. De façon similaire, l'évolution possible des réglementations sur les modes de logement des truies pose la question des relations entre comportement maternel, survie des porcelets et types de bâtiments d'élevage. Cependant, le projet ANR COSADD «Critères et Objectifs de Sélection Animale pour un Développement Durable» n'a pas permis de dégager de tendances claires pour la mise en place, dans un avenir proche, de lignées de porcs spécifiquement adaptées à ces modalités alternatives d'élevage. L'évolution envisagée des réglementations sur la pratique de la castration oblige aussi à s'interroger sur la «généricité» des résultats zootechniques actuellement disponibles dans l'optique, par exemple, de l'élevage de mâles entiers ou immunocastrés. Dans ce contexte, la question des interactions entre génotype et environnement prend une nouvelle dimension à travers le changement des génotypes exploités.

\section{Références}

Ashworth C.J., Pickard A.R., 1998. Embryo survival and prolificacy. In: Progress in pig science. Wiseman J., Varley M.A., Chadwick J.P. (Eds), Nottingham University Press, Nottingham, UK, 303-325.

Berger F., Dagorn J., Le Denmat M., Quillien J.P., Vaudelet J.C., Signoret J.P., 1997. Perinatal losses in outdoor pig breeding. A survey of factors influencing piglet mortality. Ann. Zootech., 46, 321-329.

Bidanel J.P., Ducos A., 1994. Utilisation du BLUP modèle animal pour l'évaluation génétique des porcs de race Large White et Landrace Français sur la prolificité. Journ. Rech. Porcine Fr., 26, 321-326.

Botermans J.A.M., Svendsen J., 2000. Effect of feeding environment on performance, inju- ries and behaviour in growing-finishing pigs: Group-based studies. Acta Agric. Scand., Section Anim. Sci., 50, 237-249.

Boulot S., Quesnel H., Quiniou N., 2008 Management of high prolificacy in French herds: can we alleviate side effects on piglet survival? Adv. Pork Prod., 19, 1-8.

Breuer K., Sutcliffe M.E.M., Mercer J.T., Rance K.A., O’Connell N.E., Sneddon I.A., Edwards S.A., 2005. Heritability of clinical tail-biting and its relation to performance traits. Livest. Prod. Sci., 93, 87-94.

Busch M.E., Christensen G., Wachmann H., Petersen H.H., 2007. Osteochondrosis in the elbow joint of slaughter pigs. Part 2: Connection with growth and heritability. Dansk Veterinartidsskrift, 90, 20-25.
Canario L., 2006. Aspects génétiques de la mortalité des porcelets à la naissance et en allaitement précoce : relations avec les aptitudes maternelles des truies et la vitalité des porcelets. Thèse doctorale, Génétique animale et comportement, Institut National Agronomique Paris-Grignon (INAPG), 343 p.

Canario L., Roy N., Gruand J., Bidanel J.P., 2006a. Genetic variation of farrowing kinetics traits and relationships with litter size and perinatal mortality in French Large White sows. J. Anim. Sci., 84, 1053-1058.

Canario L., Tribout T., Bidanel J. P., 2006 b. Genetic trends for reproduction and maternal abilities in French Large White pigs. 57 $7^{\text {th }}$ Ann. Meet. E.A.A.P., Antalya, Turkey, Commission on Animal Genetics, Commun. G10-01. 
Canario L., Rydhmer L., Gogué J., Tribout T., Bidanel J.P., 2007a. Estimation of genetic trends from 1977 to 1998 for farrowing characteristics in the French Large White breed using frozen semen. Animal, 1, 929-938.

Canario L., Père M.C., Tribout T., Thomas F., David C., Gogué J., Herpin P., Bidanel J.P., Le Dividich J., 2007b. Estimation of genetic trends from 1977 to 1998 of body composition and physiological state of Large White pigs at birth. Animal, 1, 1409-1413.

Canario L., Bergsma R., D'Eath R.B., Lawrence A.B., Roehe R., Lundeheim N., Rydhmer L., Knol E., Turner S.P., 2008. Genetic relations between the group effect for average daily gain, and post-mixing aggression and skin lesions in Swedish pigs. 59th Ann Meet. E.A.A.P., Vilnius, Lithuania. Book of Abstracts, 200

Canario L., Rydhmer L., Roehe R., Lundeheim N., Lawrence A., Knol E., Bergsma R., Turner S., 2010. Compétition ou coopération pour la croissance : relation génétique avec l'agressivité des porcs élevés en groupe. Journ. Rech. Porcine Fr., 42, 155-160.

Clapperton M., Bishop S.C., Cameron N.D. Glass E.J., 2005. Associations of acute phase protein levels with growth performance and with selection for growth performance in Large White pigs. Anim. Sci., 81, 213-220.

Clapperton M., Glass E.J., Bishop S.C., 2008. Pig peripheral blood mononuclear leucocyte subsets are heritable and genetically correlated with performance. Animal, 2, 1575-1584

Crawley A.M., Mallard B., Wilkie B.N. 2005. Genetic selection for high and low immune response in pigs: Effects on immunoglobulin isotype expression. Vet. Immunol. Immunop. 108, 71-76.

Désautés C., Sarrieau A., Caritez J.C., Mormède P., 1997. Behavior and pituitaryadrenal function in Large White and Meishan pigs. Dom. Anim. Endocrinol., 16, 193-205.

Devillers N., Farmer C., Le Dividich J., Prunier A., 2007. Variability of colostrum yield and colostrum intake in pigs. Animal, 1, 1033 1041

Dewey C.E., 1999. Diseases of the nervous and locomotor systems. In: Diseases of swine. Straw B., D'Allaire S., Mengeling W.L., Taylor D.J. (Eds), Ames Iowa State University Press, USA, 861-882.

Dourmad J.Y., 1988. Ingestion spontanée d'aliment chez la truie en lactation : de nombreux facteurs de variation. INRA Prod. Anim., $1,141-146$

Dourmad J.Y., Hassouna M., Robin P., Guingand N., Meunier-Salaün M.C., Lebret B. 2009 . Influence of pig rearing system on animal performance, manure composition and gaseous emissions. Animal, 3, 606-616.

Eissen J.J., Kanis E., Kemp B., 2000. Sow factors affecting voluntary feed intake during lactation. Livest. Prod. Sci., 64, 147-165.

Engblom L., Lundeheim N., Dalin A.M Andersson K., 2007. Sow removal in Swedish commercial herds. Livest. Sci., 106, 76-86.

Etienne M., Legault C., Dourmad J.Y., Noblet J., 2000. Production laitière de la truie : estimation, composition, facteurs de variation et évolution. Journ. Rech. Porcine Fr., 32, 253-264.

Flori L., Renard C., Urien C., Hu XiaoXiang, Bao-Liang Fan, Lecardonnel J.,
Ducroix-Crepy C, Piumi F., Hugot K, Bidanel J.P., Lefevre F., Rogel-Gaillard C., Chardon P., 2006. Une puce à ADN ciblée sur la région du Complexe Majeur d'Histocompatibilité chez le porc : construction et premières exploitations. Journ. Rech. Porcine Fr., 38, 119-124.

Gourdine J.L., Bidanel J.P., Noblet J., Renaudeau D. 2006. Effects of breed and season on performance of lactating sows in a tropical humid climate. J. Anim. Sci., 84, 360-369.

Guo Y.M., Ai H.S., Ren J., Wang G.J., Wen Y., Mao H.R., Lan L.T., Ma J.W., Brenig B., Rothschild M.F., Haley C.S., Huang L.S., 2009 A whole genome scan for quantitative trait loci for leg weakness and its related traits in a large F2 intercross population between White Duroc and Erhualian. J. Anim. Sci., 87, 1569-1575.

Heinonen M., Oravainen J., Orro T., SeppäLassila L., Ala-Kurikka E., Virolainen J.V., Tast A., Peltoniemi O.A.T., 2006. Lameness and fertility of sows and gilts in randomly selected loose-housed herds in Finland. Vet. Rec., 159, 383-387.

Herpin P., Le Dividich J., Amaral N., 1993. Effect of selection for lean tissue growth on body composition and physiological state of the pig at birth. J. Anim. Sci., 71, 2645-2653.

Hu Z., Reecy J.M., 2007. Animal QTLdb: Beyond a Repository. A public platform for qtl comparisons and integration with diverse types of structural genomic information. Mamm. Genome, 18, 1-4.

IFIP, Institut du Porc. Performances des élevages de porcs, http://www.ifip.asso.fr/

Ilari E., Daridan D., Desbois D., Fraysse J.L., Fraysse J., 2004. Les systèmes de production du porc en France : typologie des exploitations agricoles ayant des porcs. Journ. Rech. Porcine Fr., 36, 1-8.

Ilari E., Chatellier V., Marouby H., 2007. Les exploitations agricoles avec porcins en France face à la réforme de la PAC. Journ. Rech. Porcine Fr., 39, 167-172.

Jondreville C., Dourmad J.Y., 2005. Le phosphore dans la nutrition des porcs. In : Numéro spécial, Le phosphore dans l'alimentation animale. F. Meschy, S. Sauvant, P. Pinot (Eds). INRA Prod. Anim., 18, 183-192.

Le Dividich J., Rooke J.A., Herpin P., 2005. Nutritional and immunological importance of colostrum for the new-born pig. J. Agri. Sci., 143, 469-485.

Le Floc'h, 2000. Conséquences d'un état inflammatoire ou infectieux sur le métabolisme et le besoin en acides aminés chez le porc. INRA Prod. Anim., 13, 3-10.

Legault C., 1998. Génétique et prolificité chez la truie : la voie hyperprolifique et la voie sinoeuropéenne. INRA Prod. Anim., 11, 214-218.

Legault C., Gruand, J., 1976. Amélioration de la prolificité des truies par la création d'une lignée «hyperprolifique» et l'usage de l'insémination artificielle : principe et résultats expérimentaux préliminaires. Journ. Rech. Porcine Fr., 3, 11-17.

Lenoir H., Mercat M.J., 2008. Bilan des effectifs, des performances de reproduction et de la variabilité génétique des 6 races locales françaises. Techni Porc, 31 (3), 15-22.

Lucia T. Jr, Dial G.D., Marsh W.E., 2000. Lifetime reproductive performance in female pigs having distinct reasons for removal. Livest. Prod. Sci., 63, 213-222.
Luther H., Vögeli P., Hofer A., 2009. Increasing genetic E. coli F18 resistance in Swiss pigs. $60^{\text {th }}$ Ann. Meet. E.A.A.P., Barcelone, 208.

Martel G., Dedieu B., Dourmad J.Y., 2008. Simulation of sow herd dynamics with emphasis on performance and distribution of periodic task events. J. Agric. Sci., 146, 365-380.

Martel G., Dourmad J.Y., Dedieu B., 2009. Quelles attentes des éleveurs de truies vis-à-vis du rythme de travail au cours de la semaine et quels liens avec les pratiques d'élevage? Journ. Rech. Porcine Fr., 41, 217-224.

Merlot E., 2004. Conséquences du stress sur la fonction immunitaire chez les animaux d'élevage. INRA Prod. Anim., 17, 255-264.

Merlot E., Thomas F., Mounier A.M., Dourmad J.Y., Lebret B., Prunier B., 2010. Influence de la race et du mode de logement sur la sécrétion de cortisol, l'immunité et la santé des porcs à l'engrais. Journ. Rech. Porcine Fr., 42, 21-26.

Nakano T., Brennan J.J., Aherne F.X., 1987. Leg weakness and osteochondrosis in swine: a review. Can. J. Anim. Sci., 67, 883-901.

Nielsen B., Jørgensen C.B., Vernersen A., Fredholm M., 2009. Effect of selection for E. coli F4ab/ac resistance in pigs. $60^{\text {th }}$ Ann. Meet. E.A.A.P., Barcelone, Espagne, 208.

Noblet J., Etienne M., 1986. Effect of energy level in lactating sows on yield and composition of milk and nutrient balance of piglets. J. Anim. Sci., 63, 1888-1896.

Noblet J., Close W.H., Heavens R.P., Brown D., 1985. Studies on the energy metabolism of the pregnant sow. 1. Uterus and mammary tissue development. Brit. J. Nut., 53, 251-265.

Noblet J., Dourmad J.Y., Etienne J., 1990. Energy utilization in pregnant and lactating sows: modelling of energy requirements. J. Anim. Sci., 68, 562-572.

Norring M, Valros A., Munksgaard L., Puumala M., Kaustell K.O., Saloniemi H., 2006. The development of skin, claw and teat lesions in sows and piglets in farrowing crates with two concrete flooring materials. Acta Agric. Scand., Animal Science, 56, 148-154.

Père M.C., Etienne M., 2000. Uterine blood flow in sows: effects of pregnancy stage and litter size. Reprod. Nutr. Dev., 40, 369-382.

Père M.C., Dourmad J.Y., Etienne M., 1997. Effect of number of pig embryos in the uterus on their survival and development and on maternal metabolism. J. Anim. Sci., 75, 1337-1342.

Prunier A., Lebret B., 2009. La production biologique de porcs en France : caractéristiques des élevages, impacts sur la santé, le bien-être et la qualité des produits. In : Numéro spécial, Elevage Bio. J.M. Perez (Ed). INRA Prod. Anim., 22, 179-188.

Prunier A., Quesnel H., 2000. Nutritional influences on the hormonal control of reproduction in female pigs. Livest. Prod. Sci., 63, $1-19$.

Prunier A., Messias de Bragança M., Le Dividich J., 1997. Influence of high ambient temperature on performance of reproductive sows. Livest. Prod. Sci., 52, 123-133.

Prunier A., Heinonen M., Quesnel H., 2010. High physiological demands in intensively raised pigs: impact on health and welfare. Animal, 4, doi:10.1017/S175173111000008X. Sous presse. 
Quesnel H., 2005. Etat nutritionnel et reproduction chez la truie allaitante. INRA Prod. Anim., 18, 277-286.

Quesnel H., 2009. Nutritional and lactational effects on follicular development in the pig. Proc. VIII ${ }^{\text {th }}$ Int. Conf. Pig Reprod., Banff, Canada, June 2009, 22, abstract

Quesnel H., Brossard L., Valancogne A. Quiniou N., 2008. Influence of some sow characteristics on within-litter variation of piglet birth weight. Animal, 2, 1842-1849.

Quesnel H., Venturi E., Royer E., Elleboudt F., Boulot S., Serriere S., Martinat-Botté F., 2009. Dietary intake during early pregnancy does not influence embryonic survival and variability in gilts. Proc. VIII ${ }^{\text {th }}$ Int. Conf. Pig Reprod., Banff, Canada, June 2009, 129, abstract.

Quiniou, N., Noblet. J., 1999. Influence of high ambient temperature on performances of multiparous lactating sows. J. Anim. Sci., 77, 2121-2134.

Quiniou N., Dagorn J., Gaudré D., 2002. Variation of piglets' birth weight and consequences on subsequent performance. Livest. Prod. Sci., 78, 63-70.

Rauw W.M., Kanis E., Noordhuizen-Stassen E.N., Grommers F.J., 1998. Undesirable side effects of selection for high production efficiency in farm animals: a review. Livest. Prod. Sci., 56, 15-33.

Rose N., Le Diguerher G., Eveno E., Jolly J.P., Larour G., L'hostis A., Blanchard P., Oger A., Le Dimma M., Jestin A., Madec F., 2003. Facteurs de risque de l'expression de la maladie de l'amaigrissement du porcelet (MAP) dans les élevages de type naisseur-engraisseur en France. Journ. Rech. Porcine Fr., 35, 383 392 .

Schenck E.L., McMunn K.A., Rosenstein D.S., Stroshine R.L., Nielsen B.D., Richert B.T., Marchant-Forde J.N., Lay D.C., 2008 Exercising stall-housed gestating gilts: effects on lameness, the musculo-skeletal system, production and behaviour. J. Anim. Sci., 86, $3166-$ 3180

Shaw F.D., Trout G.R., McPhee C.P., 1995 Plasma and muscle cortisol measurements as indicators of meat quality and stress in pigs. Meat Sci., 39, 237-246.

Town S.C., Patterson J.L., Pereira C.Z Gourley G., Foxcroft G.R., 2005. Embryonic and fetal development in a commercial dam-line genotype. Anim. Reprod. Sci., 85, 301-316.

Tribout T., Caritez J.C., Gogué J., Gruand J., Billon Y., Bouffaud M., Lagant H., Le Dividich J., Thomas F., Quesnel H., Guéblez R., Bidanel J.P., 2003. Estimation, par utilisation de semence congelée, du progrès génétique réalisé en France entre 1977 et 1998 dans la race porcine Large White : résultats pour quelques caractères de reproduction femelle. Journ. Rech. Porcine Fr., 35, 285-292.

Tribout T., Caritez J.C., Gogué J., Gruand J., Bouffaud M., Billon Y., Péry C., Griffon H., Brenot S., Le Tiran M.H., Bussières F., Le Roy P., Bidanel J.P., 2004. Estimation, par utilisation de semence congelée, du progrès génétique réalisé en France entre 1977 et 1998 dans la race porcine Large White : résultats pour quelques caractères de production et de qualite de tissus gras et maigres. Journ. Rech. Porcine Fr., 36, 275-282.
Tse W.Y., Town S.C., Murdoch G.K., Novak S., Dyck M.K., Putman C.T., Foxcroft G.R., Dixon W.T., 2008. Uterine crowding in the sow affects litter sex ratio, placental development and embryonic myogenin expression in early gestation. Reprod. Fert. Dev., 20, 497-504.

Uemoto Y., Sato S., Ohnishi C., Hirose K, Kameyama K., Fukawa K., Kudo O. Kobayashi E., 2009. Quantitative trait loci for leg weakness traits in a Landrace purebred population. Anim. Sci. J., doi: 10.1111/j.17400929.2009.00713.x.

Vonnahme K.A., Wilson M.E., Foxcroft G.R., Ford S.P., 2002. Impacts on conceptus survival in a commercial swine herd. J. Anim. Sci., 80, 553-559.

Wallenbeck A., 2009. Pigs for organic production. PhD Thesis, SLU, Sweden, $85 \mathrm{p}$.

Wilkie B.N., Mallard B. 1999. Selection for high immune response: an alternative approach to animal health maintenance? Vet. Immunol. Immunop., 72, 231-235.

Wilkie B.N., Mallard B.A., 2000. Genetic aspects of health and disease resistance in pigs. In: Breeding for disease resistance in farm animals. Bishop S.C., Nicholas F.W., Owen J.B. (Eds), 2nd Edition. C.A.B. International, Wallingford, UK, 550p.

Yoshioka G., Imaeda N., Torimoto Y., Ohtani T., Hayashi K., 2004. Influence of transport stress on serum cortisol and thyroid hormones in pigs with the Halothane gene. Anim. Sci., $75,451-456$

Ytrehus B., Carlson C.S., Ekman S., 2007. Etiology and pathogenesis of osteochondrosis. Vet. Pathol., 44, 429-448.

\section{Résumé}

L'analyse de l'évolution des performances des élevages porcins français indique une amélioration nette de la productivité numérique des truies, sans qu'il y ait eu de détérioration de leur longévité, et bien que la mortalité des porcelets se soit légèrement accrue. Cette amélioration des performances s'explique à la fois par l'obtention de progrès génétiques et par la sophistication des pratiques d'élevage. La question est toutefois posée de la capacité des truies et des porcelets à faire face aux nombreux changements biologiques qui sous-tendent l'amélioration de ces performances. Chez la truie, on a ainsi pu identifier deux problèmes essentiels : la faiblesse des aplombs et la difficulté accrue des animaux à couvrir leurs besoins nutritionnels. Chez les porcelets, la question de la compétition pour les nutriments entre les individus d'une même portée, in utero et durant la lactation, se pose de façon marquée dans les grandes portées, conduisant à un accroissement de la variabilité du développement et de la mortalité. Les performances d'engraissement des porcs se sont également améliorées, mais on note toujours, pour certains caractères, un écart important entre les résultats des élevages et le potentiel génétique, ce qui semble indiquer des problèmes d'adaptation. La sensibilité des porcs aux maladies d'origine multifactorielle constitue sûrement aujourd'hui l'un des facteurs les plus limitants dans les élevages. En termes de perspectives, les questions liées à la robustesse doivent être appréhendées à la lumière des évolutions attendues des systèmes d'élevage et des réglementations. La recherche d'animaux plus autonomes, résistant mieux aux maladies et capables de valoriser des ressources alimentaires plus diversifiées et moins en compétition avec l'alimentation humaine, fait partie des pistes à envisager.

\section{Abstract}

\section{Evolution of animal performance and robustness in pig farms}

The evolution of animal performance levels on French pig farms indicates a significant improvement of sow productivity, without any degradation of their longevity but with a tendency to a slight increase in piglet mortality. This improvement in performance results both from the genetic gains realised and the improved control of the environment of swine. However, such high performance levels imply high physiological demands for the sows and piglets, and it may be questioned whether the animals are able to face these changes. In sows, the problems of leg weakness and the increased risk of occurrence of nutritional deficit during lactation were identified. With a greater prolificacy, competition among piglets for nutrients, either in utero or during lactation, is enhanced. Although on-farm performance of fattening pigs has been improved, it still remains below the genetic potential for certain traits, indicating some problems of adaptation. In this context, the sensitivity of pigs to multifactorial diseases remains an important problem. In the 
future, animal robustness has to be considered while taking into account the expected evolution of pig production systems and changes in regulations. Getting more autonomous pigs, having improved resistance to diseases and higher ability to make use of more diversified feeding resources, could be perspectives for future research.

DOURMAD J.-Y., CANARIO L., GILBERT H., MERLOT E., QUESNEL H., PRUNIER A., 2010. Évolution des performances et de la robustesse des animaux en élevage porcin. In : Robustesse, rusticité, flexibilité, plasticité, résilience... les nouveaux critères de qualité des animaux et des systèmes d'élevage. Sauvant D., Perez J.M. (Eds). Dossier Inra Prod. Anim., $23,53-64$. 